\title{
The Culture Conditions for the Mycelial Growth of Phellinus spp.
}

\author{
Woo-Sik Jo*, Young-Hyun Rew, Sung-Guk Choi, Geon-Sik Seo ${ }^{1}$, Jae-Mo Sung ${ }^{2}$ and Jae-Youl Uhm ${ }^{3}$ \\ Department of Agricultural Environment, Gyeongbuk Agricultural Technology Administration, Daegu 702-320, Korea \\ ${ }^{\prime}$ Department of Industrial Crops, Korea National Agricultural College, Hwasung 441-893, Korea \\ ${ }^{2}$ Department of Applied Bioloty, Kangwon National University, Chuncheon 200-701, Korea \\ ${ }^{3}$ Department of Agricultural Biology, Kyeongbuk National University, Daegu 702-701, Korea \\ (Received October 25, 2006)
}

\begin{abstract}
Phellinus genus belonged to Hymenochaetaceae of Basidiomycetes and has been well known as one of the most popular medicinal mushrooms due to high antitumor activity. This study was carried out to obtain the basic information for mycelial culture conditions of Phellinus linteus, $P$. baumii, and $P$. gilvus. According to colony diameter and mycelial density, the media for suitable mycelial growth of them were shown in MEA, glucose peptone, and MCM. The optimum temperature for mycelial growth was $30^{\circ} \mathrm{C}$. Carbon and nitrogen sources were mannose and malt extract, respectively. The optimum $\mathrm{C} / \mathrm{N}$ ratio was $10: 1$ to $5: 1$ with $2 \%$ glucose concentration, vitamin was thiamine-HCl, organic acid was succinic acid, and mineral salt was $\mathrm{MgSO}_{4} 7 \mathrm{H}_{2} \mathrm{O}$.
\end{abstract}

KEYWORDS: Culture condition, Medicinal mushroom, Phellinus baumii, Phellinus gilvus, Phellinus linteus

The number of mushrooms on Earth is estimated at 140,000 , yet maybe only $10 \%$ (approximately 14,000 named species) are known (Kirk et al., 2001). For a long time, mushrooms have been valued as an edible and medicinal resource. Phellinus genus is known about 220 species and is found mainly in tropical America and Africa (Dai et al., 1998). The genus is distributed into 7 species and commonly referred to as Sangwhang in Korea (Lee, 1993; Hong, 2000). Many kinds of Phellinus spp. (e.g. P. linteus, P. igniarius, P. gilvus, P. pini and P. hartigii) are known and they have a variety of medicinal effects (Lee et al., 1996). Among them P. linteus, $P$. baumii and $P$. gilvus have been known as ones of the most popular medicinal mushrooms due to their high antitumor activity in east Asia (Ikekawa et al., 1968; Han et al., 1999; Bae et al., 2004), and safety of acute oral toxicity test (Han et al., 2001). P. linteus, P. baumii and P. gilvus have been cultivated by mushroom farmers in Korea. $P$. gilvus is cheaper than P. linteus and P. baumii because of very short cultivating period. Therefore, it has a possibility which can be developed as a functional food and livestock for industrial application in near future. This study was focused on culture conditions affecting the optimal mycelial growth of $P$. linteus, $P$. baumii and $P$. gilvus.

\section{Materials and Methods}

Fungal isolates. The isolates of Phellinus species used in this study were listed in Table 1. P. linteus ASI 26099, P. baumii Nongong and P. gilvus KCTC 6653 were pre-

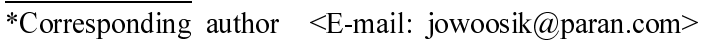

sented by Rural Development Administration, Nongong Agricultural Product Company, and Biological Resources Center of Korea, respectively. All isolates were maintained on Potato Dextrose Agar medium (PDA).

Culture media and temperature: Twelve different culture media were prepared to screen suitable culture media to mycelial growth of $P$. linteus, $P$. baumii and $P$. gilvus (Table 2). The culture media were sterilized for 20 minutes at $121^{\circ} \mathrm{C}$ and aseptically poured into plastic petridish. An inoculum was removed from seven days old cultures of Phellinus spp. grown on PDA at $30^{\circ} \mathrm{C}$. Mycelial disk ( $5 \mathrm{~mm}$ in diameter) from the cultures was placed in the center of each $85 \mathrm{~mm}$ plastic petridishs containing about $20 \mathrm{ml}$ of 12 different media. The fungi were incubated under the dark condition for 9 days at $30^{\circ} \mathrm{C}$. Thereafter the mycelial growth, density and color of the colony were examined. To screen temperature condition for a suitable growth of Phellinus spp. The ranges of temperature were $10^{\circ} \mathrm{C}, 15^{\circ} \mathrm{C}, 20^{\circ} \mathrm{C}, 25^{\circ} \mathrm{C}, 30^{\circ} \mathrm{C}$ and $35^{\circ} \mathrm{C}$, respectively. The fungi cultured on Malt Extract Agar (MEA) for 10 days by the same method above. Then the measurement of mycelial growth was performed.

\section{Effect of favorable nutrient sources.}

Carbon sources: The experiment was performed on the mushroom minimal media (MMM: dextrose $20 \mathrm{~g}, \mathrm{MgSO}_{4}$ $0.5 \mathrm{~g}, \mathrm{KH}_{2} \mathrm{PO}_{4} 0.46 \mathrm{~g}, \mathrm{~K}_{2} \mathrm{HPO}_{4} 1 \mathrm{~g}$, asparagine $2 \mathrm{~g}$, thiamine- $\mathrm{HCl} 120 \mu \mathrm{g}$, agar $20 \mathrm{~g}$, DW $1,000 \mathrm{ml}$ ) supplemented with each of 10 carbon sources. Each carbon source was added to mushroom minimal media at the concentration of $2 \%$. The fungi were incubated under the dark condition for 10 days at $30^{\circ} \mathrm{C}$. Thereafter we exam- 
Table 1. List of Phellinus spp. strains used in this study

\begin{tabular}{llcll}
\hline \hline Scientific name & Strain name & Korean common name & Origin culture & Organ \\
\hline Phellinus linteus & Koryosanghwang & 목질진훍버섯 & ASI 26099 & Rural Development Administration, Korea \\
Phellinus baumii & Jangsusanghwang & 장수상황버섯 & Nongong & Nongong Agricultural Product Co., Korea \\
Phellinus gilvus & Hwanggeumsanghwang & 마른진훍버섯 & KCTC 6653 & Biological Resources Center, Korea \\
\hline
\end{tabular}

Table 2. Composition of media used in this study

\begin{tabular}{|c|c|c|c|c|c|c|c|c|c|c|c|c|}
\hline \multirow{2}{*}{$\begin{array}{l}\text { Nutritional } \\
\text { reagents }\end{array}$} & \multicolumn{12}{|c|}{ Media and composition $(\mathrm{g} / \mathrm{l})$} \\
\hline & PDA & MEA & YEA & $\begin{array}{c}\text { Czapek } \\
\text { dox }\end{array}$ & $\begin{array}{l}\text { Glucose } \\
\text { peptone }\end{array}$ & YMA & $\begin{array}{c}\text { Malt yeast } \\
\text { extract }\end{array}$ & Leonian & MCM & Henner-berg & Lilly & Hoppkins \\
\hline Glucose & & & 10 & & 10 & & 10 & 25 & 20 & 50 & & 10 \\
\hline Sucrose & & & & 30 & & & & & & & & \\
\hline Maltose & & & & & & & & & & & 10 & \\
\hline Peptone & & 3 & & & 10 & 5 & & & 2 & & & \\
\hline Yeast extract & & & 5 & & 10 & 3 & 5 & & 2 & & & \\
\hline Malt extract & & 30 & & & 15 & 3 & 3 & & & & & \\
\hline Potato extract & 4 & & & & & & & & & & & \\
\hline DL-Asparagine & & & & & & & & & & & 2 & \\
\hline Dextrose & 20 & & & & & 10 & & & & & & \\
\hline $\mathrm{NaNO}_{3}$ & & & & 3 & & & & & & 2 & & \\
\hline $\mathrm{MgSO}_{4} \cdot 7 \mathrm{H}_{2} \mathrm{O}$ & & & & 0.5 & & & & 0.5 & 0.5 & 0.5 & 0.5 & 0.5 \\
\hline $\mathrm{KCl}$ & & & & 0.5 & & & & & & & & \\
\hline $\mathrm{FeSO}_{4} \cdot 7 \mathrm{H}_{2} \mathrm{O}$ & & & & 0.01 & & & & 0.02 & & & & \\
\hline $\mathrm{CaCl}_{2} \cdot 2 \mathrm{H}_{2} \mathrm{O}$ & & & & & & & & & & 0.1 & & \\
\hline $\mathrm{ZnSO}_{4} \cdot 7 \mathrm{H}_{2} \mathrm{O}$ & & & & & & & & & & & & \\
\hline $\mathrm{MnSO}_{4} \cdot 5 \mathrm{H}_{2} \mathrm{O}$ & & & & & & & & 0.01 & & & & \\
\hline $\mathrm{K}_{2} \mathrm{HPO}_{4}$ & & & & 1 & & & & & 1 & & & \\
\hline $\mathrm{KH}_{2} \mathrm{PO}_{4}$ & & & & 1 & & & & 1 & 0.5 & 1 & 1 & 0.1 \\
\hline $\mathrm{KNO}_{3}$ & & & & & & & & & & 2 & & 2 \\
\hline Agar & 15 & 15 & 20 & 20 & 20 & 20 & 20 & 20 & 20 & 20 & 20 & 20 \\
\hline
\end{tabular}

ined the mycelial growth, density and color of the colony. Nitrogen sources: To screen nitrogen source suitable to the mycelial growth of Phellinus spp., the experiment was performed on the mushroom minimal media supplemented with each of 12 nitrogen sources. Each nitrogen source was added to mushroom minimal media at the concentration of $0.2 \%$. A $5 \mathrm{~mm}$ diameter plug an inoculum of Phellinus spp. cultures placed in the centure of petridish and incubated under the dark condition for 10 days at $30^{\circ} \mathrm{C}$. Thereafter the mycelial growth, density and color of the colony were examined.

C/N ratio: On the mushroom minimal media which were mixed with $10,8,6,4,2,1,0.4$ and $0.2 \%$ glucose as a carbon source and then mixed continually with $0.2 \%$ $\mathrm{NaNO}_{3}$ as a nitrogen source, the mycelial growth of Phellinus spp. was exammined. The $\mathrm{C} / \mathrm{N}$ ratio was adjusted to $50: 1,40: 1,30: 1,20: 1,10: 1,5: 1,2: 1$ and $1: 1$ in each medium. Inoculated each media incubated under the dark condition for 9 days at $30^{\circ} \mathrm{C}$. Thereafter the mycelial growth, density and color of the colony were examined.

Vitamin: On the sterialized mushroom minimal media which were mixed with thiamine- $\mathrm{HCl} 0.1 \mathrm{mg} / \mathrm{l}$, riboflavin
$0.5 \mathrm{mg} / \mathrm{l}$, biotine $0.005 \mathrm{mg} / \mathrm{l}$, pyridoxine $0.5 \mathrm{mg} / \mathrm{l}$ and nicotinamide $2.0 \mathrm{mg} / \mathrm{l}$ those were filtrated by metrical membrane filter $(0.2 \mu \mathrm{m})$. Inoculated each media incubated under the dark condition for 9 days at $30^{\circ} \mathrm{C}$. Thereafter the mycelial growth, density and color of the colony were examined.

Organic acid: On the mushroom minimal media which were mixed with acetic acid, citric acid, maleic acid, lactic acid, succinic acid and fumaric acid at the concentration of $0.1 \%$, respectively. Inoculated each media incubated under the dark condition for 9 days at $30^{\circ} \mathrm{C}$. Thereafter the mycelial growth, density and color of the colony were examined.

Mineral salt: To screen mineral salts suitable to the mycelial growth of Phellinus spp., the experiment was performed on the YM solid media (peptone $5 \mathrm{~g}$, yeast extract $3 \mathrm{~g}$, malt extract $3 \mathrm{~g}$, dextrose $10 \mathrm{~g}$, agar $20 \mathrm{~g}$ and DW 1,000 $\mathrm{ml}$ ) eleminated mineral salt which was supplemented with each of 9 mineral salts. Each mineral salt was added to YM solid media at the concentration of $0.1 \%$. Inoculated each media incubated under the dark condition for 9 days at $30^{\circ} \mathrm{C}$. Thereafter the mycelial growth, density and color of the colony were examined. 
Table 3. Effect of culture medium on mycelial growth of Phellinus spp. at $30^{\circ} \mathrm{C}$

\begin{tabular}{|c|c|c|c|c|c|c|c|c|c|}
\hline \multirow{2}{*}{ Culture media } & \multicolumn{3}{|c|}{ Colony diameter (mm/9 days) } & \multicolumn{3}{|c|}{ (Mycelial density ${ }^{\text {a) }}$} & \multicolumn{3}{|c|}{ (Color ${ }^{\text {b) }}$} \\
\hline & P. $l$ & $P . b$ & P. $g$ & P. $l$ & P. $b$ & P. $g$ & P. $l$ & P. $b$ & P. $g^{\mathrm{c})}$ \\
\hline PDA & $7.7 \pm 1.53^{\mathrm{f}(\mathrm{z})}$ & $68.7 \pm 1.15^{\mathrm{de}}$ & $57.3 \pm 2.51^{\mathrm{b}}$ & ST & $\mathrm{C}$ & $\mathrm{SC}$ & SY & Y & $\mathrm{Br}$ \\
\hline MEA & $35.0 \pm 0^{c}$ & $77.0 \pm 1.73^{\text {ab }}$ & $45.7 \pm 3.51^{\text {cd }}$ & $\mathrm{SC}$ & $\mathrm{C}$ & $\mathrm{SC}$ & SY & Y & $\mathrm{Br}$ \\
\hline YEA & $27.3 \pm 0.58^{d}$ & $55.0 \pm 1.0^{\mathrm{g}}$ & $59.7 \pm 1.53^{\mathrm{b}}$ & ST & $\mathrm{SC}$ & ST & SY & Y & $\mathrm{Br}$ \\
\hline Czapek dox & $21.7 \pm 1.53^{\mathrm{e}}$ & $38.7 \pm 3.1^{\mathrm{h}}$ & $12.7 \pm 2.51^{\mathrm{e}}$ & $\mathrm{T}$ & $\mathrm{T}$ & $\mathrm{T}$ & $\mathrm{W}$ & W & $\mathrm{W}$ \\
\hline Glucose peptone & $47.0 \pm 1.73^{\mathrm{b}}$ & $73.7 \pm 1.15^{\mathrm{bcd}}$ & $39.3 \pm 2.08^{d}$ & $\mathrm{SC}$ & $\mathrm{C}$ & ST & SY & $\mathrm{Y}$ & $\mathrm{Br}$ \\
\hline YMA & $46.0 \pm 1.0^{\mathrm{b}}$ & $70.0 \pm 2.0^{\mathrm{cd}}$ & $56.3 \pm 6.02^{\mathrm{bc}}$ & $\mathrm{SC}$ & $\mathrm{C}$ & ST & SY & $\mathrm{Y}$ & $\mathrm{Br}$ \\
\hline Malt yeast extract & $47.3 \pm 0.58^{\mathrm{b}}$ & $62.7 \pm 2.51^{\mathrm{f}}$ & $58.3 \pm 2.88^{\mathrm{b}}$ & $\mathrm{SC}$ & $\mathrm{C}$ & $\mathrm{SC}$ & SY & $\mathrm{Y}$ & $\mathrm{Br}$ \\
\hline Leonian & $52.0 \pm 1.0^{\mathrm{a}}$ & $80.3 \pm 0.57^{\mathrm{a}}$ & $39.3 \pm 1.15^{\mathrm{d}}$ & $\mathrm{T}$ & $\mathrm{SC}$ & $\mathrm{T}$ & $\mathrm{W}$ & Y & $\mathrm{W}$ \\
\hline MCM & $52.0 \pm 1.0^{\mathrm{a}}$ & $64.3 \pm 1.53^{\mathrm{ef}}$ & $65.7 \pm 2.98^{\text {ab }}$ & $\mathrm{SC}$ & $\mathrm{C}$ & $\mathrm{SC}$ & SY & Y & $\mathrm{Br}$ \\
\hline Hennerberg & $46.0 \pm 3.46^{\mathrm{b}}$ & $73.0 \pm 1.0^{\text {bcd }}$ & $40.7 \pm 1.15^{\mathrm{d}}$ & $\mathrm{T}$ & $\mathrm{SC}$ & $\mathrm{T}$ & W & $\mathrm{Y}$ & W \\
\hline Lilly & $49.9 \pm 1.53^{\mathrm{ab}}$ & $72.7 \pm 2.51^{\mathrm{bcd}}$ & $72.3 \pm 2.51^{\mathrm{a}}$ & $\mathrm{SC}$ & $\mathrm{C}$ & $\mathrm{T}$ & SY & $\mathrm{Y}$ & $\mathrm{W}$ \\
\hline Hoppkins & $52.7 \pm 1.15^{\mathrm{a}}$ & $74.0 \pm 1.73^{\mathrm{bc}}$ & $60.3 \pm 4.04^{b}$ & $\mathrm{~T}$ & $\mathrm{ST}$ & $\mathrm{T}$ & $\mathrm{W}$ & $\mathrm{Y}$ & W \\
\hline
\end{tabular}

a): C; compact, SC; somewhat compact, ST; somewhat thin, T; thin

b): Br; brownish, SY; somewhat Yellowish, Y; Yellowish, W; Whitish

c): P. l; P. linteus ASI 26099, P. b; P. baumii Nongong, P. g; P. gilvus KCTC 6653

z): Values in the same line with different literal differ at Duncan's multiple range test $(\mathrm{P}<0.05)$ and results are mean \pm standard error of three replicates.

\section{Results and Discussion}

Screening of the suitable culture media. The mycelial growth of Phellinus spp. was favorable in MEA, glucose peptone, and MCM whereas was poor in Czapek dox, Leonian, Hennerberg and Hoppkins medium (Table 3). Lee et al. (2004) reported that the mycelial growth of $P$. linteus was favorable in MYA and SMS medium. Chi et al. (1996) reported that the mycelial growth of $P$. linteus was favorable in YM, malt yeast extract, and MCM medium whereas was poor in Czapek dox, Leonian, Lilly, Modified Lutz and Hoppkins medium. We concluded that the above results were similar with this study. The mycelial growth of $P$. linteus isolate ASI 26099 was less than P. baumii isolate Nongong

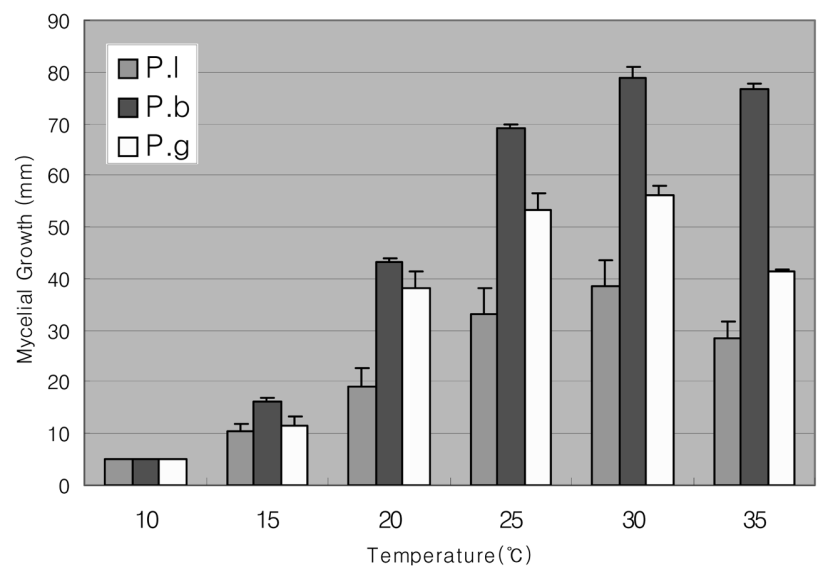

Fig. 1. Mycelial growth of $P$. linteus ASI $26099(P . l), P$. baumii Nongong $(P . b)$, and $P$. gilvus KCTC 6653 ( $P$. $g)$ on MEA for 10 days at different temperatures. Vertical bars show standard errors $(n=3)$. and $P$. gilvus isolate KCTC 6653. Colony's color was that $P$. linteus isolate ASI 26099 was light yellow, $P$. baumii isolate Nongong was yellow and $P$. gilvus isolate KCTC 6653 was brown.

Effect of the temperature: The suitable temperature for the mycelial growth of Phellinus spp. was obtained at $30^{\circ} \mathrm{C}$ (Fig. 1). Their mycelial growth was suppressed rapidly at the temperature higher than $30^{\circ} \mathrm{C}$ and lower than $20^{\circ} \mathrm{C}$. Heo et al. (2004) reported that the optimum culture temperature of $P$. baumii and $P$. igniarius was $25 \sim 30^{\circ} \mathrm{C}$, Chi et al. (1996) reported the optimum culture temperature of $P$. linteus was $25 \sim 30^{\circ} \mathrm{C}$, Rew et al. (2000) reported that the optimum culture temperature of $P$. gilvus was $25 \sim 30^{\circ} \mathrm{C}$. It was concluded that the above results were similar with this study.

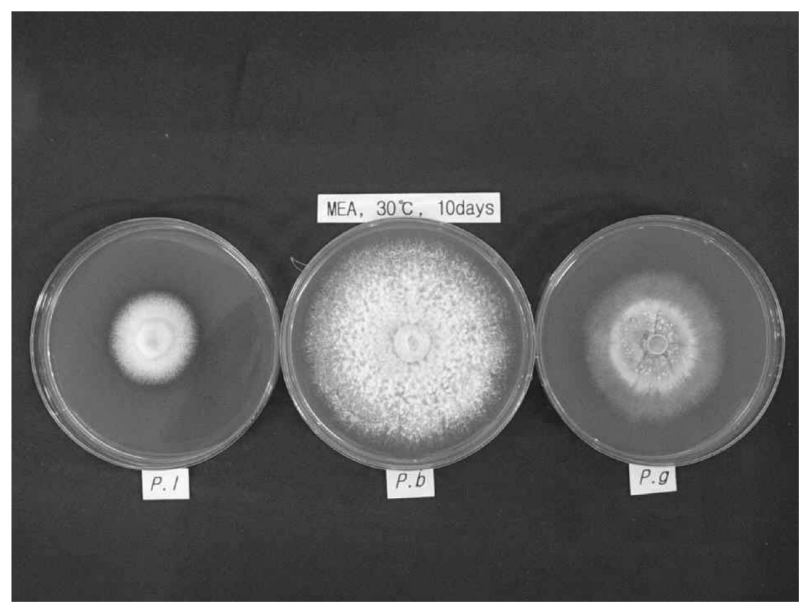

Fig. 2. Colonies of P. linteus ASI 26099 (P. l), P. baumii Nongong $(P . b)$, and $P$. gilvus KCTC $6653(P . g)$ grown on MEA medium for 10 days at $30^{\circ} \mathrm{C}$. 
Table 4. Effect of carbon source on the mycelial growth of Phellinus spp.

\begin{tabular}{|c|c|c|c|c|c|c|c|c|c|}
\hline \multirow{2}{*}{ Culture media } & \multicolumn{3}{|c|}{ Colony diameter $(\mathrm{mm} / 10$ days $)$} & \multicolumn{3}{|c|}{ Mycelial density $^{\text {a) }}$} & \multicolumn{3}{|c|}{ (Color ${ }^{\text {b) }}$} \\
\hline & P. $l$ & P. $b$ & P. $g$ & P. $l$ & P. $b$ & P. $g$ & P. $l$ & $P . b$ & P. $g^{\mathrm{c})}$ \\
\hline Sucrose & $55.3 \pm 5.03^{\mathrm{bc}(z)}$ & $72.3 \pm 2.52^{b-e}$ & $83.7 \pm 1.53^{\mathrm{a}}$ & ST & $\mathrm{SC}$ & ST & W & Y & $\mathrm{Br}$ \\
\hline Lactose & $51.3 \pm 1.53^{\mathrm{c}}$ & $66.7 \pm 2.89^{\mathrm{e}}$ & $84.7 \pm 0.58^{\mathrm{a}}$ & ST & $\mathrm{SC}$ & ST & W & Y & $\mathrm{W}$ \\
\hline Dextrin & $63.0 \pm 2.65^{\mathrm{ab}}$ & $72.7 \pm 3.06^{\mathrm{bcd}}$ & $85.3 \pm 0.58^{\mathrm{a}}$ & ST & $\mathrm{SC}$ & ST & $\mathrm{W}$ & Y & Y \\
\hline Mannitol & $64.0 \pm 3.04^{\mathrm{ab}}$ & $76.3 \pm 1.53^{\mathrm{abc}}$ & $82.3 \pm 2.08^{\mathrm{a}}$ & ST & $\mathrm{C}$ & $\mathrm{SC}$ & $\mathrm{W}$ & Y & $\mathrm{Br}$ \\
\hline Maltose & $61.3 \pm 3.51^{\mathrm{abc}}$ & $79.3 \pm 1.15^{\mathrm{de}}$ & $82.7 \pm 3.21^{\mathrm{a}}$ & SC & $\mathrm{C}$ & $\mathrm{SC}$ & $\mathrm{W}$ & $\mathrm{Y}$ & $\mathrm{Br}$ \\
\hline Glucose & $57.7 \pm 3.21^{\mathrm{abc}}$ & $74.0 \pm 1.73^{\mathrm{bcd}}$ & $79.7 \pm 1.53^{\mathrm{a}}$ & $\mathrm{SC}$ & $\mathrm{C}$ & $\mathrm{C}$ & W & $\mathrm{Y}$ & $\mathrm{Br}$ \\
\hline Fructose & $63.3 \pm 1.53^{\mathrm{ab}}$ & $81.7 \pm 1.53^{\mathrm{a}}$ & $84.7 \pm 0.58^{\mathrm{a}}$ & $\mathrm{SC}$ & $\mathrm{C}$ & $\mathrm{C}$ & W & $\mathrm{Y}$ & $\mathrm{Br}$ \\
\hline Sorbitol & $57.3 \pm 2.52^{\mathrm{abc}}$ & $77.3 \pm 2.08^{\mathrm{ab}}$ & $63.0 \pm 4.09^{\mathrm{b}}$ & $\mathrm{SC}$ & $\mathrm{C}$ & $\mathrm{SC}$ & $\mathrm{W}$ & Y & $\mathrm{Br}$ \\
\hline Mannose & $65.7 \pm 1.53^{\mathrm{a}}$ & $77.7 \pm 1.53^{\mathrm{ab}}$ & $84.3 \pm 1.15^{\mathrm{a}}$ & $\mathrm{C}$ & $\mathrm{C}$ & $\mathrm{C}$ & $\mathrm{W}$ & Y & $\mathrm{Br}$ \\
\hline Starch & $66.0 \pm 1.73^{\mathrm{a}}$ & $71.0 \pm 1.73^{\text {cde }}$ & $84.0 \pm 1.73^{\mathrm{a}}$ & $\mathrm{SC}$ & $\mathrm{C}$ & $\mathrm{C}$ & $\mathrm{W}$ & Y & $\mathrm{Br}$ \\
\hline
\end{tabular}

a): $\mathrm{C}$; compact, $\mathrm{SC}$; somewhat compact, ST; somewhat thin, T; thin

b): Br; brownish, SY; somewhat Yellowish, Y; Yellowish, W; Whitish

c): P. l; P. linteus ASI 26099, P. b; P. baumii Nongong, P. g; P. gilvus KCTC 6653

z): Values in the same line with different literal differ at Duncan's multiple range test $(\mathrm{P}<0.05)$ and results are mean \pm standard error of three replicates.

Table 5. Effect of nitrogen source on the mycelial growth of Phellinus spp.

\begin{tabular}{|c|c|c|c|c|c|c|c|c|c|}
\hline \multirow{2}{*}{ Culture media } & \multicolumn{3}{|c|}{ Colony diameter $(\mathrm{mm} / 10$ days $)$} & \multicolumn{3}{|c|}{ Mycelial density ${ }^{\text {a) }}$} & \multicolumn{3}{|c|}{ (Color ${ }^{\text {b) }}$} \\
\hline & P. $l$ & P. $b$ & P. $g$ & P. $l$ & P. $b$ & P. $g$ & P. $l$ & P. $b$ & P. $g^{\mathrm{c})}$ \\
\hline Yeast extract & $57.3 \pm 2.08^{\mathrm{ab}(\mathrm{z})}$ & $59.0 \pm 3.61^{\circ}$ & $75.0 \pm 4.35^{\mathrm{bc}}$ & $\mathrm{SC}$ & $\mathrm{SC}$ & SC & W & SY & $\mathrm{Br}$ \\
\hline Malt extract & $62.3 \pm 2.52^{\mathrm{a}}$ & $77.7 \pm 0.58^{\mathrm{a}}$ & $83.3 \pm 2.89^{\mathrm{a}}$ & $\mathrm{SC}$ & $\mathrm{C}$ & $\mathrm{C}$ & $\mathrm{W}$ & $\mathrm{Y}$ & $\mathrm{Br}$ \\
\hline Peptone & $61.3 \pm 1.53^{\mathrm{ab}}$ & $78.7 \pm 1.53^{\mathrm{a}}$ & $84.7 \pm 0.58^{\mathrm{a}}$ & $\mathrm{SC}$ & $\mathrm{C}$ & $\mathrm{C}$ & SY & Y & $\mathrm{Br}$ \\
\hline Urea & $14.7 \pm 2.51^{\mathrm{g}}$ & $48.3 \pm 5.03^{\mathrm{d}}$ & $15.3 \pm 0.58^{\mathrm{f}}$ & $\mathrm{T}$ & ST & $\mathrm{T}$ & $\mathrm{W}$ & SY & $\mathrm{W}$ \\
\hline Ammonium nitrate & $55.3 \pm 1.15^{\mathrm{bc}}$ & $76.7 \pm 1.53^{\text {ab }}$ & $79.3 \pm 1.53^{\text {ab }}$ & $\mathrm{SC}$ & $\mathrm{C}$ & $\mathrm{SC}$ & SY & $\mathrm{Y}$ & $\mathrm{W}$ \\
\hline Ammonium chloride & $49.0 \pm 2.00^{\mathrm{d}}$ & $77.3 \pm 1.15^{\mathrm{a}}$ & $64.7 \pm 5.03^{\mathrm{d}}$ & $\mathrm{SC}$ & $\mathrm{C}$ & $\mathrm{SC}$ & SY & $\mathrm{Y}$ & $\mathrm{Br}$ \\
\hline Ammonium acetate & $42.3 \pm 3.21^{\circ}$ & $69.7 \pm 3.21^{\mathrm{b}}$ & $33.3 \pm 2.08^{\mathrm{e}}$ & ST & $\mathrm{SC}$ & ST & $\mathrm{W}$ & SY & $\mathrm{Br}$ \\
\hline Ammonium sulpate & $49.3 \pm 2.08^{\text {cd }}$ & $78.7 \pm 1.15^{\mathrm{a}}$ & $67.3 \pm 4.93^{\text {cd }}$ & $\mathrm{SC}$ & $\mathrm{C}$ & $\mathrm{SC}$ & $\mathrm{W}$ & $\mathrm{Y}$ & $\mathrm{Br}$ \\
\hline Potassium nitrate & $59.7 \pm 1.53^{\mathrm{ab}}$ & $75.3 \pm 1.53^{\text {ab }}$ & $82.3 \pm 2.52^{\mathrm{ab}}$ & $\mathrm{SC}$ & $\mathrm{C}$ & $\mathrm{C}$ & $\mathrm{W}$ & $\mathrm{Y}$ & $\mathrm{Br}$ \\
\hline Sodium nitrate & $56.3 \pm 1.15^{\mathrm{ab}}$ & $79.3 \pm 1.15^{\mathrm{a}}$ & $81.7 \pm 3.21^{\mathrm{ab}}$ & $\mathrm{SC}$ & $\mathrm{C}$ & $\mathrm{C}$ & $\mathrm{W}$ & Y & $\mathrm{Br}$ \\
\hline Calcium nitrate & $48.3 \pm 2.08^{\mathrm{de}}$ & $78.7 \pm 2.31^{\mathrm{a}}$ & $81.3 \pm 1.53^{\mathrm{ab}}$ & $\mathrm{SC}$ & $\mathrm{C}$ & $\mathrm{SC}$ & $\mathrm{Y}$ & $\mathrm{Y}$ & $\mathrm{Br}$ \\
\hline L-glutamic acid & $29.7 \pm 1.53^{\mathrm{f}}$ & $74.0 \pm 3.61^{\mathrm{ab}}$ & $79.7 \pm 0.58^{\mathrm{ab}}$ & $\mathrm{SC}$ & $\mathrm{C}$ & $\mathrm{C}$ & $\mathrm{Y}$ & Y & $\mathrm{Br}$ \\
\hline L-arginine & $62.0 \pm 3.61^{\mathrm{a}}$ & $80.0 \pm 2.00^{\mathrm{a}}$ & $79.3 \pm 1.15^{\mathrm{ab}}$ & $\mathrm{SC}$ & $\mathrm{C}$ & $\mathrm{C}$ & $\mathrm{W}$ & $\mathrm{Y}$ & $\mathrm{Br}$ \\
\hline
\end{tabular}

a): C; compact, SC; somewhat compact, ST; somewhat thin, T; thin

b): Br; brownish, SY; somewhat Yellowish, Y; Yellowish, W; Whitish

c): P. l; P. linteus ASI 26099, P. b; P. baumii Nongong, P. g; P. gilvus KCTC 6653

z): Values in the same line with different literal differ at Duncan's multiple range test $(\mathrm{P}<0.05)$ and results are mean \pm standard error of three replicates.

\section{Effect of favorable nutrient sources.}

Carbon sources: The carbon source promoting a mycelial growth and mycelial density of Phellinus spp. was glucose and mannose (Table 4). Among 10 carbon sources, mannose showed colony diameter of $P$. gilvus isolate KCTC 6653 was $84 \mathrm{~mm}$. The mycelial density of $P$. gilvus isolate KCTC 6653 was compact in glucose. Chi et al. (1996) reported that the optimum culture carbon sources of $P$. linteus were glucose, mannose and dextrose.

Nitrogen sources: The nitrogen source promoting a mycelial growth of Phellinus spp. was malt extract, peptone and potassium nitrate (Table 5). The mycelial density of $P$. baumii isolate Nongong was compact in malt extract. Among 13 nitrogen sources, malt extract showed colony diameter of $P$. baumii isolate Nongong was $78 \mathrm{~mm}$. Chi et al. (1996) reported that the optimum culture nitrogen sources of $P$. linteus were cassamino acid, alanine and glutamic acid.

C/N ratio: On the culture media which were mixed with $2 \%$ glucose as carbon source and then adjusted to the $\mathrm{C} / \mathrm{N}$ ratio of $10: 1$ and $5: 1$, Phellinus spp. showed the most favorable mycelial growth (Table 6). Lee et al. (2004) reported that the optimum culture $\mathrm{C} / \mathrm{N}$ ratio of $P$. linteus was $10: 1$. Also our results were similar to those of Chi et al. (1996).

Vitamin: When various vitamins were added to the MMM medium, thiamine- $\mathrm{HCl}$ and biotine were very 
Table 6. Effect of $\mathrm{C} / \mathrm{N}$ ratio on the mycelial growth of Phellinus spp.

\begin{tabular}{|c|c|c|c|c|c|c|c|c|c|}
\hline \multirow{2}{*}{ Culture media } & \multicolumn{3}{|c|}{ Colony diameter (mm/9 days) } & \multicolumn{3}{|c|}{ Mycelial density ${ }^{\text {a) }}$} & \multicolumn{3}{|c|}{ Color $^{\mathrm{b})}$} \\
\hline & P. $l$ & $P . b$ & P. $g$ & P. $l$ & P. $b$ & P. $g$ & P. $l$ & P. $b$ & P. $g^{\mathrm{c})}$ \\
\hline $50: 1$ & $41.7 \pm 0.58^{\mathrm{c}(z)}$ & $45.7 \pm 1.53^{\mathrm{e}}$ & $25.7 \pm 1.53^{\mathrm{d}}$ & $\mathrm{SC}$ & $\mathrm{SC}$ & ST & SY & Y & $\mathrm{Br}$ \\
\hline $40: 1$ & $49.0 \pm 1.00^{\mathrm{ab}}$ & $54.7 \pm 3.51^{\mathrm{d}}$ & $38.7 \pm 3.06^{\circ}$ & $\mathrm{C}$ & $\mathrm{C}$ & ST & SY & $\mathrm{Y}$ & $\mathrm{Br}$ \\
\hline $30: 1$ & $47.3 \pm 1.53^{\mathrm{b}}$ & $58.7 \pm 1.53^{\text {cd }}$ & $54.7 \pm 3.06^{\mathrm{b}}$ & $\mathrm{C}$ & $\mathrm{C}$ & $\mathrm{SC}$ & SY & $\mathrm{Y}$ & $\mathrm{Br}$ \\
\hline $20: 1$ & $50.7 \pm 0.58^{\mathrm{ab}}$ & $62.3 \pm 4.04^{\mathrm{bc}}$ & $55.3 \pm 2.52^{\mathrm{b}}$ & $\mathrm{SC}$ & $\mathrm{C}$ & $\mathrm{SC}$ & SY & $\mathrm{Y}$ & $\mathrm{Br}$ \\
\hline $10: 1$ & $52.7 \pm 2.52^{\mathrm{a}}$ & $72.3 \pm 2.08^{\mathrm{a}}$ & $63.3 \pm 3.51^{\mathrm{ab}}$ & $\mathrm{SC}$ & $\mathrm{C}$ & $\mathrm{SC}$ & SY & Y & $\mathrm{Br}$ \\
\hline $5: 1$ & $51.0 \pm 2.00^{\mathrm{ab}}$ & $72.0 \pm 2.00^{\mathrm{a}}$ & $66.3 \pm 1.53^{\mathrm{a}}$ & $\mathrm{SC}$ & $\mathrm{C}$ & $\mathrm{SC}$ & SY & Y & $\mathrm{Br}$ \\
\hline $2: 1$ & $48.7 \pm 1.53^{\mathrm{ab}}$ & $68.7 \pm 2.31^{\mathrm{ab}}$ & $68.6 \pm 2.52^{\mathrm{a}}$ & $\mathrm{SC}$ & $\mathrm{SC}$ & ST & SY & $\mathrm{Y}$ & $\mathrm{Br}$ \\
\hline $1: 1$ & $42.7 \pm 2.52^{\mathrm{c}}$ & $62.3 \pm 3.06^{\mathrm{bc}}$ & $64.7 \pm 1.53^{\text {ab }}$ & ST & $\mathrm{SC}$ & ST & SY & $\mathrm{Y}$ & $\mathrm{Br}$ \\
\hline
\end{tabular}

a): $\mathrm{C}$; compact, $\mathrm{SC}$; somewhat compact, ST; somewhat thin, $\mathrm{T}$; thin

b): Br; brownish, SY; somewhat Yellowish, Y; Yellowish, W; Whitish

c): P. l; P. linteus ASI 26099, P. b; P. baumii Nongong, P. g; P. gilvus KCTC 6653

z): Values in the same line with different literal differ at Duncan's multiple range test $(\mathrm{P}<0.05)$ and results are mean \pm standard error of three replicates.

Table 7. Effect of vitamins on the mycelial growth of Phellinus spp.

\begin{tabular}{|c|c|c|c|c|c|c|c|c|c|}
\hline \multirow{2}{*}{ Culture media } & \multicolumn{3}{|c|}{ Colony diameter (mm/9 days) } & \multicolumn{3}{|c|}{ Mycelial density ${ }^{\text {a) }}$} & \multicolumn{3}{|c|}{ Color $^{\mathrm{b})}$} \\
\hline & P. $l$ & P. $b$ & P. $g$ & P. $l$ & P. $b$ & P. $g$ & P. $l$ & P. $b$ & P. $g^{\mathrm{c})}$ \\
\hline Thiamine-HCl & $54.3 \pm 2.08^{\mathrm{a}(\mathrm{z})}$ & $61.7 \pm 1.15^{\mathrm{a}}$ & $76.7 \pm 3.06^{\text {ab }}$ & $\mathrm{C}$ & $\mathrm{C}$ & $\mathrm{C}$ & W & $\mathrm{Y}$ & $\mathrm{Br}$ \\
\hline Riboflavin & $45.3 \pm 1.53^{\mathrm{c}}$ & $48.3 \pm 3.51^{\mathrm{b}}$ & $69.3 \pm 1.15^{\circ}$ & $\mathrm{SC}$ & $\mathrm{SC}$ & $\mathrm{SC}$ & $\mathrm{W}$ & SY & $\mathrm{Br}$ \\
\hline Biotin & $51.3 \pm 2.31^{\mathrm{ab}}$ & $66.0 \pm 2.65^{\mathrm{a}}$ & $82.3 \pm 0.58^{\mathrm{a}}$ & $\mathrm{SC}$ & $\mathrm{C}$ & $\mathrm{C}$ & $\mathrm{W}$ & $\mathrm{Y}$ & $\mathrm{Br}$ \\
\hline Pyridoxine & $47.7 \pm 2.08^{\mathrm{bc}}$ & $53.7 \pm 2.89^{b}$ & $70.7 \pm 0.58^{\mathrm{bc}}$ & $\mathrm{SC}$ & $\mathrm{SC}$ & $\mathrm{SC}$ & $\mathrm{W}$ & SY & $\mathrm{Br}$ \\
\hline Nicotinamide & $38.7 \pm 1.53^{\mathrm{d}}$ & $48.0 \pm 2.65^{\mathrm{b}}$ & $60.7 \pm 3.06^{d}$ & ST & SC & SC & W & SY & $\mathrm{Br}$ \\
\hline
\end{tabular}

a): C; compact, SC; somewhat compact, ST; somewhat thin, T; thin

b): Br; brownish, SY; somewhat Yellowish, Y; Yellowish, W; Whitish

c): P. l; P. linteus ASI 26099, P. b; P. baumii Nongong, P. g; P. gilvus KCTC 6653

z): Values in the same line with different literal differ at Duncan's multiple range test $(\mathrm{P}<0.05)$ and results are mean \pm standard error of three replicates.

Table 8. Effect of organic acid on the mycelial growth of Phellinus spp.

\begin{tabular}{|c|c|c|c|c|c|c|c|c|c|}
\hline \multirow{2}{*}{ Culture media } & \multicolumn{3}{|c|}{ Colony diameter (mm/9 days) } & \multicolumn{3}{|c|}{ Mycelial density $^{\text {a) }}$} & \multicolumn{3}{|c|}{ Color $^{\text {b) }}$} \\
\hline & P. $l$ & P. $b$ & P. $g$ & P. $l$ & P. $b$ & P. $g$ & P. $l$ & P. $b$ & P. $g^{\mathrm{c})}$ \\
\hline Acetic acid & $5.0 \pm 0.0^{\mathrm{e}(z)}$ & $5.0 \pm 0.0^{\mathrm{e}}$ & $5.0 \pm 0.0^{\circ}$ & - & - & - & - & - & - \\
\hline Citric acid & $29.3 \pm 0.58^{\mathrm{bc}}$ & $71.7 \pm 2.89^{b}$ & $53.7 \pm 3.06^{\circ}$ & $\mathrm{SC}$ & $\mathrm{C}$ & $\mathrm{C}$ & Y & Y & $\mathrm{Br}$ \\
\hline Maleic acid & $16.7 \pm 1.53^{\mathrm{d}}$ & $45.7 \pm 1.53^{\mathrm{d}}$ & $30.7 \pm 1.53^{\mathrm{d}}$ & ST & SC & ST & Y & Y & $\mathrm{Br}$ \\
\hline Lactic acid & $33.7 \pm 2.08^{b}$ & $79.3 \pm 1.15^{\mathrm{a}}$ & $63.3 \pm 4.16^{\mathrm{b}}$ & $\mathrm{SC}$ & $\mathrm{C}$ & $\mathrm{SC}$ & $\mathrm{Y}$ & $\mathrm{Y}$ & $\mathrm{Br}$ \\
\hline Succinic acid & $48.7 \pm 1.15^{\mathrm{a}}$ & $64.3 \pm 2.08^{\mathrm{c}}$ & $79.3 \pm 0.58^{\mathrm{a}}$ & $\mathrm{C}$ & $\mathrm{SC}$ & $\mathrm{C}$ & SY & $\mathrm{Y}$ & $\mathrm{Br}$ \\
\hline Fumaric acid & $24.3 \pm 4.16^{\mathrm{c}}$ & $68.3 \pm 1.53^{\mathrm{bc}}$ & $51.7 \pm 2.89^{\mathrm{c}}$ & $\mathrm{SC}$ & $\mathrm{C}$ & $\mathrm{SC}$ & $\mathrm{Y}$ & Y & $\mathrm{Br}$ \\
\hline
\end{tabular}

a): C; compact, SC; somewhat compact, ST; somewhat thin, T; thin

b): Br; brownish, SY; somewhat Yellowish, Y; Yellowish, W; Whitish

c): P. l; P. linteus ASI 26099, P. b; P. baumii Nongong, P. g; P. gilvus KCTC 6653

z): Values in the same line with different literal differ at Duncan's multiple range test $(\mathrm{P}<0.05)$ and results are mean \pm standard error of three replicates.

excellent for a mycelial growth of Phellinus spp. (Table 7). After 9 days cultivation, colony diameter of $P$. gilvus isolate $\mathrm{KCTC} 6653$ for thiamine- $\mathrm{HCl}$ and biotine were $77 \mathrm{~mm}$ and $82 \mathrm{~mm}$, respectively. Chi et al. (1996) reported that the optimum culture vitamins of $P$. linteus were biotin and $\mathrm{Ca}$-pantothenic.

Organic acid: When various organic acids were added to the MMM medium, succinic acid and lactic acid were very excellent for a mycelial growth of Phellinus spp., whereas acetic acid was no growth of Phellinus spp. (Table 8, Fig. 3). After 9 days cultivation, colony diameter of $P$. baumii isolate Nongong for succinic acid and lactic acid were $64 \mathrm{~mm}$ and $79 \mathrm{~mm}$, respectively.

Mineral salt: When various mineral salts were added to the $\mathrm{YM}$ medium, $\mathrm{MgSO}_{4} \cdot 7 \mathrm{H}_{2} \mathrm{O}$ and $\mathrm{KH}_{2} \mathrm{PO}_{4}$ were very excellent for a mycelial growth of Phellinus spp., whereas 
Table 9. Effect of mineral salt on the mycelial growth of Phellinus spp.

\begin{tabular}{|c|c|c|c|c|c|c|c|c|c|}
\hline \multirow{2}{*}{ Culture media } & \multicolumn{3}{|c|}{ Colony diameter (mm/9 days) } & \multicolumn{3}{|c|}{ Mycelial density $^{\text {a) }}$} & \multicolumn{3}{|c|}{ Color $^{\text {b) }}$} \\
\hline & P. $l$ & P. $b$ & P. $g$ & P. $l$ & P. $b$ & P. $g$ & P. $l$ & P. $b$ & P. $g^{\mathrm{c})}$ \\
\hline $\mathrm{MgSO}_{4} \cdot 7 \mathrm{H}_{2} \mathrm{O}$ & $52.3 \pm 2.08^{\mathrm{a}(z)}$ & $76.3 \pm 1.15^{\mathrm{a}}$ & $71.3 \pm 1.53^{\mathrm{a}}$ & $\mathrm{SC}$ & $\mathrm{C}$ & $\mathrm{SC}$ & SY & $\mathrm{Y}$ & $\mathrm{Br}$ \\
\hline $\mathrm{KCl}$ & $45.3 \pm 1.15^{\mathrm{bc}}$ & $67.7 \pm 4.62^{\mathrm{b}}$ & $52.7 \pm 1.15^{\mathrm{bc}}$ & $\mathrm{SC}$ & $\mathrm{C}$ & $\mathrm{SC}$ & SY & $\mathrm{Y}$ & $\mathrm{Br}$ \\
\hline $\mathrm{KH}_{2} \mathrm{PO}_{4}$ & $45.7 \pm 2.52^{b}$ & $75.3 \pm 0.58^{\mathrm{a}}$ & $58.7 \pm 2.08^{b}$ & $\mathrm{SC}$ & $\mathrm{C}$ & $\mathrm{SC}$ & SY & $\mathrm{Y}$ & $\mathrm{Br}$ \\
\hline $\mathrm{K}_{2} \mathrm{HPO}_{4}$ & $48.3 \pm 2.08^{\mathrm{ab}}$ & $60.3 \pm 2.52^{\circ}$ & $43.3 \pm 1.53^{\mathrm{d}}$ & $\mathrm{SC}$ & $\mathrm{SC}$ & $\mathrm{SC}$ & SY & Y & $\mathrm{Br}$ \\
\hline $\mathrm{NaCl}$ & $41.3 \pm 2.31^{\circ}$ & $75.3 \pm 0.58^{\mathrm{a}}$ & $45.7 \pm 2.08^{\text {cd }}$ & ST & $\mathrm{C}$ & $\mathrm{SC}$ & SY & Y & $\mathrm{Br}$ \\
\hline $\mathrm{ZnSO}_{4} \cdot 7 \mathrm{H}_{2} \mathrm{O}$ & $6.7 \pm 1.53^{\mathrm{d}}$ & $7.3 \pm 1.53^{\mathrm{f}}$ & $9.7 \pm 1.53^{\mathrm{f}}$ & $\mathrm{T}$ & $\mathrm{T}$ & $\mathrm{T}$ & SY & $\mathrm{Y}$ & $\mathrm{Br}$ \\
\hline $\mathrm{FeSO}_{4} \cdot 7 \mathrm{H}_{2} \mathrm{O}$ & $7.3 \pm 0.58^{d}$ & $13.7 \pm 1.15^{\circ}$ & $21.7 \pm 1.15^{\circ}$ & $\mathrm{T}$ & ST & ST & SY & Y & $\mathrm{Br}$ \\
\hline $\mathrm{CuSO}_{4} \cdot 5 \mathrm{H}_{2} \mathrm{O}$ & $6.0 \pm 1.00^{\mathrm{d}}$ & $32.7 \pm 2.52^{\mathrm{d}}$ & $23.3 \pm 3.51^{\mathrm{e}}$ & $\mathrm{T}$ & ST & ST & SY & Y & $\mathrm{Br}$ \\
\hline Control & $44.7 \pm 0.58^{\mathrm{bc}}$ & $64.3 \pm 2.08^{\mathrm{bc}}$ & $48.3 \pm 3.21^{\text {cd }}$ & $\mathrm{SC}$ & $\mathrm{SC}$ & $\mathrm{SC}$ & $\mathrm{Y}$ & $\mathrm{Y}$ & $\mathrm{Br}$ \\
\hline
\end{tabular}

a): C; compact, SC; somewhat compact, ST; somewhat thin, T; thin

b): Br; brownish, SY; somewhat Yellowish, Y; Yellowish, W; Whitish

c): P. l; P. linteus ASI 26099, P. b; P. baumii Nongong, P. g; P. gilvus KCTC 6653

z): Values in the same line with different literal differ at Duncan's multiple range test $(\mathrm{P}<0.05)$ and results are mean \pm standard error of three replicates.

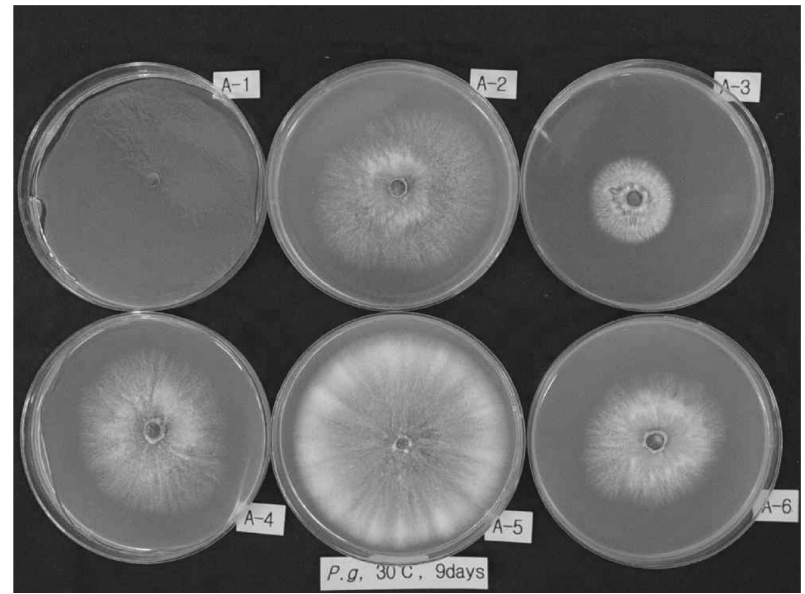

Fig. 3. Mycelial growth of P. gilvus KCTC 6653 on the mushroom minimal medium with different organic acid source A-1: Acetic acid, A-2: Citric acid, A-3: Maleic acid, A-4: Lactic acid, A-5: Succinic acid, A-6: Fumaric acid.

$\mathrm{ZnSO}_{4} \cdot 7 \mathrm{H}_{2} \mathrm{O}$ was almost no growth of Phellinus spp. (Table 9). Chi et al. (1996) reported that the optimum culture mineral salt of $P$. linteus was $\mathrm{MgSO}_{4} \cdot 7 \mathrm{H}_{2} \mathrm{O}$.

\section{Acknowledgement}

This study was supported by Technology Development Program for Agricultural and Forestry, Ministry of Agricultural and Forestry, Republic of Korea.

\section{References}

Bae, J. S., Hwang, M. H., Jang, K. H., Rhee, M. H., Lee, K. W., Jo, W. S., Choi, S. K., Yun, H. I., Lim, J. H., Kim, J. C. and Park, S. C. 2004. Comparative antitumor activity of water extracts from fruiting body of Phellinus linteus, Phellinus baumii and Phellinus gilvus. J. Toxicol. Pub. Health. 20: 37-42.

Chi, J. H., Ha, T. M., Kim, Y. H. and Rho, Y. D. 1996. Studies on the main factors affecting the mycelial growth of Phellinus linteus. Kor. J. Mycol. 24: 214-222.

Dai, Y. C. and $\mathrm{Xu}$, M. Q. 1998. Studies on the medicinal polypore, Phellinus baumii and its kin, P. linteus. Mycotaxon. 67: 191-200.

Han, S. B., Lee, C. W., Jeon, Y. J., Hong, N. D., Yoo, I. D., Yang, K. H. and Kim, H. M. 1999. The inhibitory effect of Polysaccharides isolated from Phellinus linteus on tumor growth and metastasis. Immunopharmacology 41: 157-164.

Han, Y. S., Park, S. Y., Choi, B. K. and Choung, S. Y. 2001. Acute oral toxicity studies of extract of sanghwang mushroom (Phellinus linteus). J. Applied Pharmaco. 9: 46-50.

Heo, B. S., Lee, K. S., Park, S. C. and Lee, Y. S. 2004. Cultural Conditions for the Mycelial Growth of Phellinus spp. Kor. J. Mycol. 32: 134-137.

Hong, I. P. 2000. Character of Phellinus spp. \& Production of Phellinus spp. Fruitbody. Korea Mushroom Research Society. 4: 1-15.

Ikekawa, T., Nakanishi, M., Uehara, N., Chihara, G. and Fukuoka, F. 1968. Antitumor action of some basidiomycetes, especially Phellinus linteus. Gann. 59: 155-157.

Kirk, P. M., Cannon, P. F., David, J. C. and Stalpers, J. A. 2001. Ainsworth and Bisby's dictionary of the fungi. 9th edn., CAB International, Wallingford.

Lee, J. Y. 1993. Colured Korean Mushrooms. Academy Press, Seoul, Korea.

Lee, J. H., Cho, S. M., Kim, H. M., Hong, N. D. and Yoo, I. D. 1996. Immunostimulating activity of polysaccharides from mycelia of Phellinus linteus grown under different culture conditions. J. Microbiol. 6: 52-55.

Lee, W. H., Kim, S. Y., Park, Y. J., Kim, T. W., Kim, H. K. and Sung, J. M. 2004. Favorable Conditions for Myelial Growth of Phellinus linteus. Kor. J. Mycol. 32: 95-100.

Rew, Y. H., Jo, W. S., Jeong, K. C., Yoon, J. T. and Choi, B. S. 2000. Cultural characteristics and fruitbody formation of Phellinus gilvus. Kor. J. Mycol. 28: 6-10. 